\title{
AVALIAÇÃO DA ATIVIDADE MIOELÉTRICA DO TRATO GASTROINTESTINAL EM CÃES. AVALIAÇÃO DE UM SISTEMA DE FIXAÇÃO DE ELETRODOS NA PAREDE ABDOMINAL ${ }^{1}$
}

\author{
Álvaro Antônio Bandeira Ferraz ${ }^{2}$ \\ Antônio Roberto de Barros Coelho ${ }^{2}$ \\ Cristiano de Souza Leão ${ }^{3}$ \\ Josemberg Marins Campos ${ }^{3}$ \\ Renato Dornelas Câmara Neto ${ }^{2}$ \\ Tércio Souto Bacelar ${ }^{2}$
}

\begin{abstract}
Ferraz AAB, Coelho ARB, Leão CS, Campos JM, Câmra Neto RD, Bacelar TS. Avaliação da atividade mioelétrica do trato gastrointestinal em cães: avaliação de um sistema de fixação de eletrodos na parede abdominal. Acta Cir Bras [serial online] 2002 Nov-Dez;17(6). Disponível em URL: http://www.scielo.br/acb.
\end{abstract}

RESUMO - Objetivo: A implantação de eletrodos intra-abdominais para captura de sinais de onda elétrica constitui instrumento de estudo da atividade mioelétrica do tubo gastrointestinal. $\mathrm{O}$ deslocamento destes eletrodos do local de implantação ocorre com certa freqüência em animais de experimentação não anestesiados, devido aos movimentos da musculatura diafragmática, abdominal, do peristaltismo gastrintestinal e sobretudo aos hábitos inerentes à espécie de animal estudada, particularmente presentes em caninos. No referido estudo foi proposto um procedimento que proporciona estabilidade ao referido sistema utilizando-se recursos simples e de custos irrelevantes. Métodos: Os autores apresentam através de diagramas e fotos um sistema de ancoração dos eletrodos elétricos na pele dos animais com botões. Resultados: Foram realizados 5 experimentos, fixandose em cada animal 3 eletrodos bipolares. Os animais foram mantidos em repouso até a completa recuperação do íleo paralítico. Não foi identificado nos animais estudados nenhuma migração dos eletrodos. Conclusão: O procedimento proposto é eficiente, simples, de fácil confecção e permite o monitoramento da atividade mioelétrica, em cães não anestesiados, por períodos de tempo prolongado.

DESCRITORES - Motilidade gastrointestinal. Eletromiografia. Atividade elétrica. Eletrodos. Cirurgia experimental.

\section{INTRODUÇÃO}

A implantação de eletrodos intra-abdominais para captura de sinais de onda elétrica constitui instrumento de estudo da atividade mioelétrica do tubo gastrointestinal ${ }^{1,2,3,4}$. O deslocamento destes eletrodos do local de implantação ocorre com certa freqüência em animais de experimentação não anestesiados, devido aos movimentos da musculatura diafragmática, abdominal, do peristaltismo gastrintestinal e sobretudo aos hábitos inerentes à espécie de animal estudada (lambeduras, mordeduras etc...), particularmente presentes em caninos. Assim, seria desejável prover a integridade do sistema de condução do impulso elétrico, mantendo-se

1. Trabalho realizado no Núcleo de Cirurgia Experimental do Depto. de Cirurgia - Centro de Ciências da Saúde - Hospital das Clínicas - UFPE.

2. Professor Adjunto, Doutor, Depto. de Cirurgia - Centro de Ciências da Saúde- UFPE.

3. Aluno do Programa de Pós-Graduação em Cirurgia - Centro de Ciências da Saúde - UFPE. 
folga no trajeto intraperitoneal dos fios condutores, provendo-se fixação efetiva dos mesmos na parede abdominal, assim como dificultando-se o acesso do animal à parte externa do circuito.

Dispositivos com estas finalidades, tendo como característica a eficiência associada a maior grau de complexidade e custos mais elevados, foram descritos e estão representados na Figura $1^{5}$.

Desse modo, durante estudo da atividade mioelétrica do estomago em cães, utilizando-se recursos simples e de custos irrelevantes, foi proposto um procedimento que proporciona estabilidade ao referido sistema.

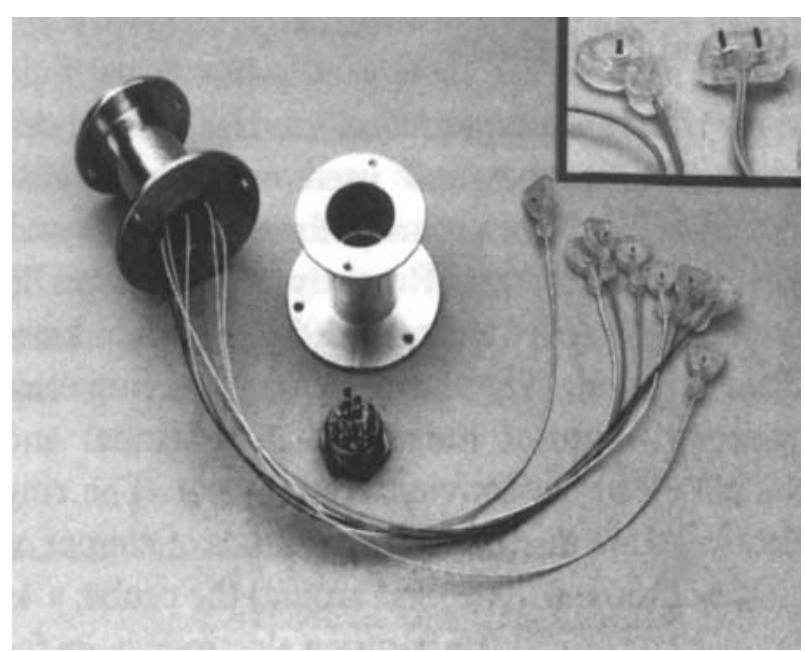

FIGURA 1 - Figura de um sistema de fixação dos eletrodos no trato gastrointestinal de animais de experimentação.

\section{MÉTODOS}

Após a implantação e fixação de eletrodos bipolares (fio de marcapasso cardíaco) na camada seromuscular da grande curvatura do estomago, os fios condutores, deixados com suficiente folga entre o estomago e o peritônio, foram passados através da parede abdominal, utilizando-se a ponta agulhada dos mesmos e exteriorizando-os através de um dos orifícios de botões de vestuário de quatro furos. Em seguida os fios foram trazidos novamente para a cavidade abdominal através de um segundo orifício dos botões e passados de volta, através da parede abdominal, no sentido da pele, exteriorizando-se os mesmos por um terceiro orifício (Figura $2 \mathrm{~A}$ ).

A complementação do procedimento foi realizada, enovelando-se o restante dos fios condutores sob os botões de vestuário. A parte agulhada dos eletrodos, aquelas que seriam conectadas ao sistema de captação e amplificação dos sinais, foram então introduzidas sob as alças formadas pelos fios condutores, sobre os botões de vestuário (Figura $2 \mathrm{~B}$ ).
A

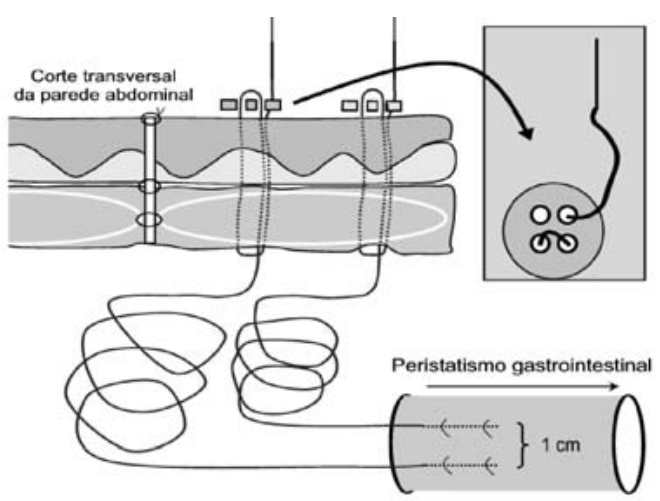

B

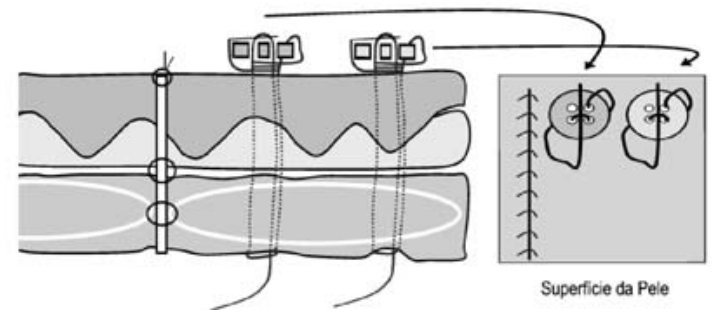

FIGURA 2 - Desenho esquemático do processo proposto. A. Implantação seromuscular dos eletrodos no estômago, trajeto amplo percorrido pelos fios condutores até o peritônio, dupla passagem dos fios pela parede abdominal com ancoração dos mesmos através de dois orifícios de botões de vestuário de quatro furos e exterioriza' ao da extremidade agulhada desses fios por um terceiro orifício. B. Enovelamento do restante dos fios condutores sob os botões de vestuário e introdução da parte agulhada dos eletrodos entre as alças formadas pelos fios condutores e os botões.

O uso de botões de vestuário de cores variadas permite a identificação dos vários pares de eletrodos posicionados no segmento gastrointestinal. O processo permite a ancoração dos fios condutores na parede abdominal, ao nível da pele, utilizando-se a característica rígida dos botões de vestuário, de modo que a tração exercida sobre as extremidades externas dos mesmos tende a otimizar o mecanismo de ancoração (Figura 3).

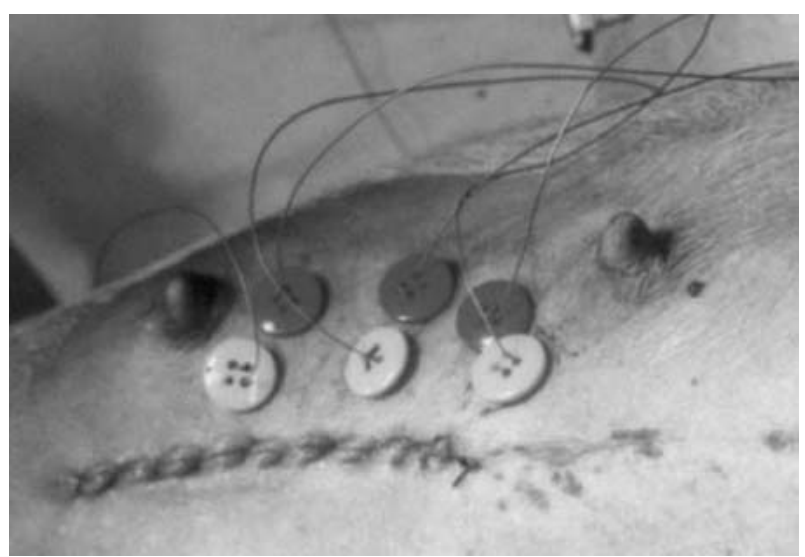

FIGURA 3 - Sistema de ancoração dos eletrodos gastrointestinais com a utilização de botões. 
Um curativo foi realizado sobre a sutura abdominal e os dispositivos de ancoração (botões de vestuário), contidos em seguida por vestimenta tubular completa semielástica de "cotton", com finalidade de dificultar a ação do animal sobre os dispositivos instalados sobre a pele (Figura 4).

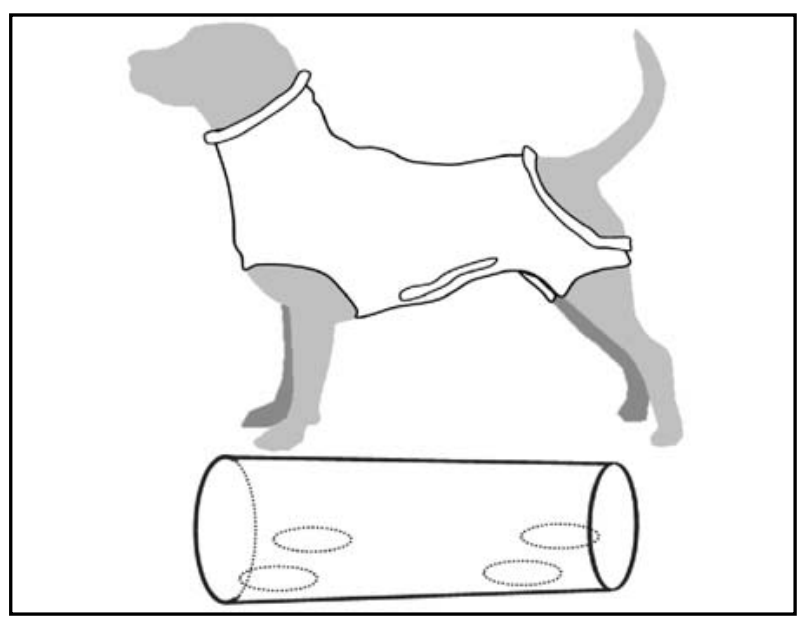

FIGURA 4 - Vestimenta tubular semielástica de "cotton" com objetivo de contenção do curativo, assim como de proteção dos dispositivos instalados sobre a pele contra a ação dos animais.

\section{RESULTADOS}

O deslocamento de eletrodos na cavidade abdominal determina um registro típico e de fácil reconhecimento (Figura 5).

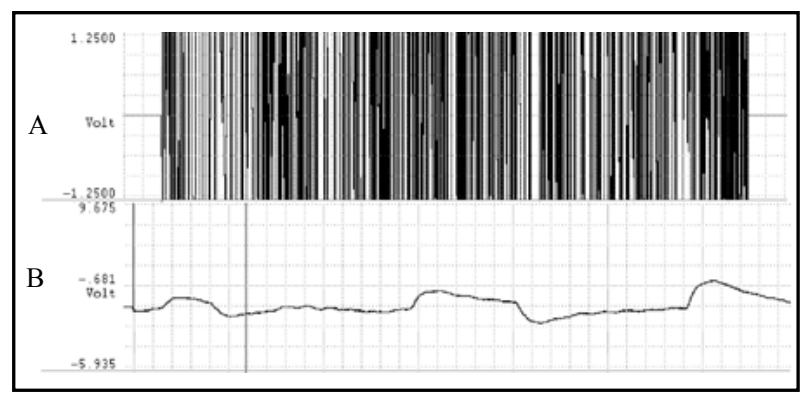

FIGURA 5 - A. Traçado indicando deslocamento do eletrodo. B. Traçado revelando atividade mioelétrica no estômago.
Em cinco cães foram implantados 3 eletrodos bipolares na camada seromuscular do corpo gástrico.

Após a recuperação do íleo paralítico (3 dias de pós-operatótio), foram realizados um período de aquisição de dados de 60 minutos denominado, período controle. Após este período foram realizadas mais 90 minutos de aquisição da atividade mioelétrica agora com o animal se alimentando com dieta padronizada.

Não foi detectado deslocamentos dos eletrodos nos 5 animais estudados.

\section{CONCLUSÃO}

O estudo da atividade mioelétrica do trato gastrointestinal em animais, e particularmente no cão, requer um complexo e trabalhoso sistema de manutenção e fixação dos eletrodos. A confecção de sistemas como o exposto na figura 1 requer o envolvimento dos eletrodos em silicone e a fixação em um recipiente de metal, que requer tecnologia e mão de obra especializada.

O procedimento proposto é eficiente, simples, de fácil confecção e permite o monitoramento da atividade mioelétrica, em cães não anestesiados, por períodos de tempo prolongado.

\section{REFERÊNCIAS}

1. Ferraz AAB, Cowles VE, Condon RE, Sschulte WJ. Opioid and non opioid analgesic drug effects on cólon contractions in monkeys. Dig. Dis. Sci., 1995;40(7):1417-9.

2. Ferraz ÁAB. Atividade mioelétrica do cólon: avaliação experimental da recuperação do íleo paralítico pós-operatório após colectomia convencional e laparoscópica [Tese Doutorado]. Universidade Federal de Pernambuco - Centro de Ciências Saúde; 1995.

3. Frantzides CT, Condon RE, Cowles V. Early postoperative colon electrical response activity. Surg Forum 1985;36:163-5.

4. Frantzides CT, Condon RE, Schulte WJ, Cowles V. Effects of morphine on colonic mioeletric and motor activity in subhuman primates. Am J Physiol 1990;21:G247-252.

5. Soper NJ, Sarr MG. Electromyography. In. Kumar D, Gustavsson S. An illustrated guide to gastrointestinal motility. London: John Wiley \& Sons Ltd.; 1988. p 101-12.

6. Falcão SC. Estudo experimental sobre o uso da pele de rã (Rana catesbeiana) como curativo biológico oclusivo em feridas cutâneas produzidas em cães [Tese - Mestrado]. Universidade Federal Rural de Pernambuco; 1999. 
Ferraz AAB, Coelho ARB, Leão CS, Campos JM, Câmra Neto RD, Bacelar TS. Gastrointestinal myoelectric activity in dogs: evaluation of a fixation system of electrodes on the abdominal wall. Acta Cir Bras [serial online] 2002 Nov-Dec;17(6). Available from URL: http://www.scielo.br/acb.

ABSTRACT - Objective: The use of intra-abdominal electrodes in the study of gastrointestinal electrical wave is an important instrument of the gastrointestinal mioelectric activity. However, the dislocations of the electrodes in non-anaesthetized animals due to diaphragmatic muscle movements, gastrointestinal movements and mainly due to the animals habits, especially in dogs. The study has the objective to demonstrate a procedure that stabilize the system using simple resources an irrelevant cost. Methods: The authors demonstrate under diagrams and picture a fixed system to fix the electrical electrodes through the animal skin using bottoms. Results: The system was used in 5 animals. Each animal had 3 bipolar electrodes. During the study it was not identified any electrode migration. The animals was kept resting until the complete recovery of the postoperative ileus. Conclusion: The procedure proposed is efficient, simple, and easy to be made and allow an acquisition of the mioelectric activity, in dogs not anaesthetized, for long periods.

KEY WORDS - Gastrointestinal motility. Electromiography. Electrical activity. Electrodes. Experimental surgery.

Conflito de interesse: nenhum

Fonte de financiamento: nenhuma

Endereço para correspondência:

Álvaro Antônio Bandeira Ferraz

Av. Beira Rio, 240/2501

50750-400 Recife - PE

aabf@truenet.com.br

Data do recebimento: 02/10/2002

Data da revisão: 19/10/2002

Data da aprovação: 03/11/2002

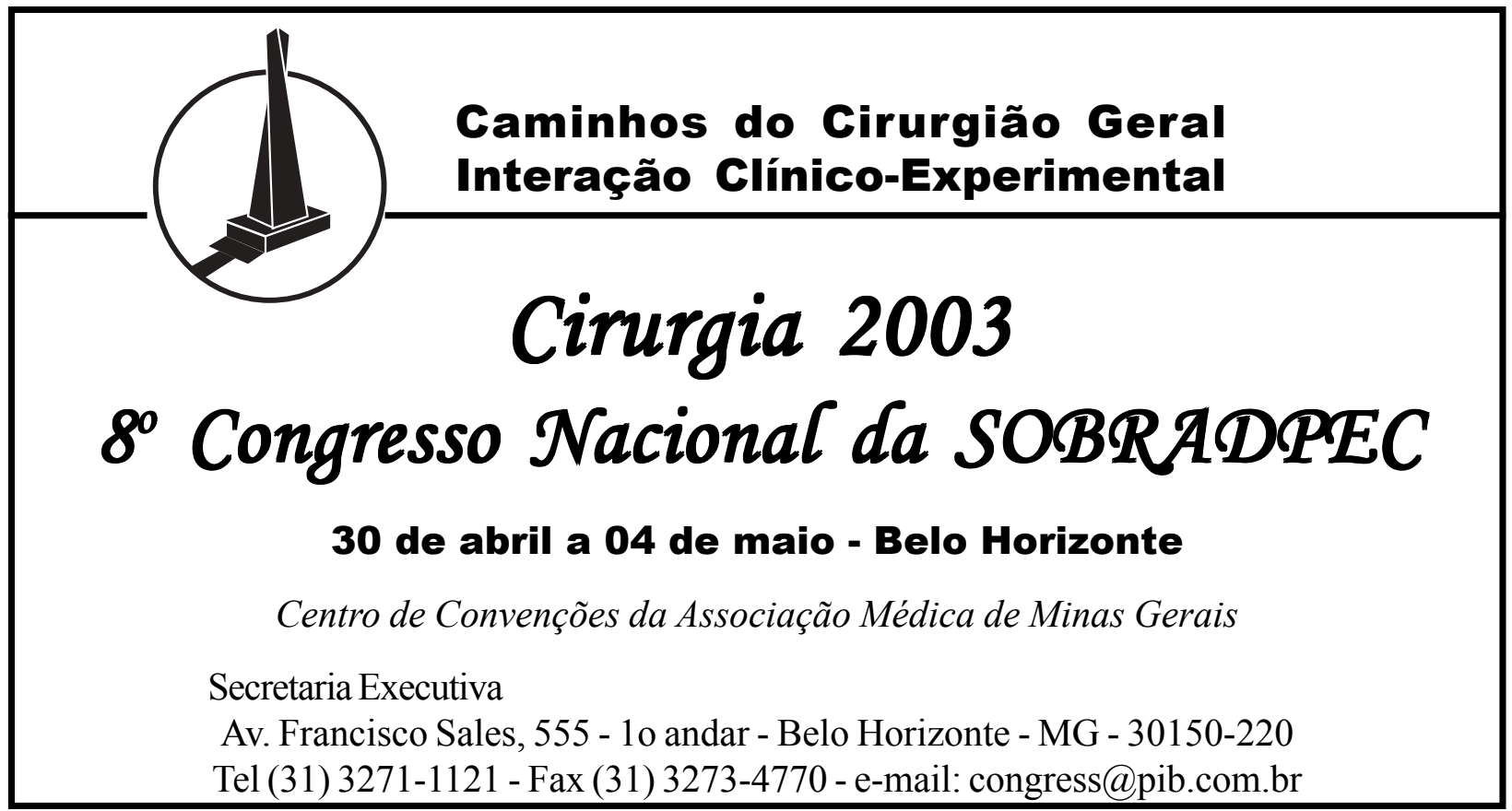

